\title{
Data report: elemental, isotopic, and Rock-Eval compositions of bulk sediments from Hole U1357A in the Adélie Basin, Antarctica'
}

\author{
Gee Soo Kong ${ }^{2}$ and Robert B. Dunbar ${ }^{3}$
}

\begin{abstract}
Chapter contents

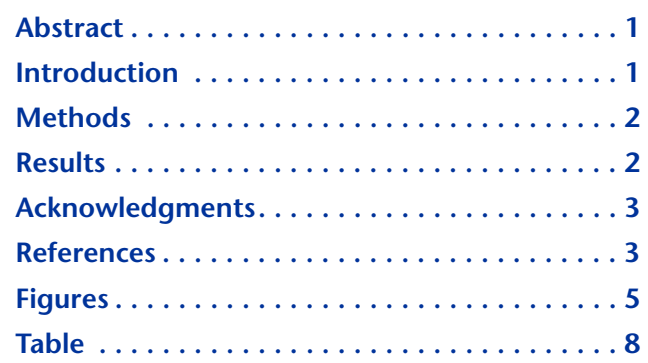

${ }^{1}$ Kong, G.S., and Dunbar, R.B., 2014. Data report: elemental, isotopic, and Rock-Eval compositions of bulk sediments from Hole U1357A in the Adélie Basin, Antarctica. In Escutia, C., Brinkhuis, H., Klaus, A., and the Expedition 318 Scientists, Proc. IODP, 318: Tokyo (Integrated Ocean Drilling Program Management International, Inc.). doi:10.2204/iodp.proc.318.201.2014 2 Petroleum and Marine Research Division, Korea Institute of Geoscience and Mineral Resources, 92 Gwahang-no, Yuseong-gu, Daejeon 305-350, Korea. kong@kigam.re.kr

${ }^{3}$ Department of Geological and Environmental Sciences, Stanford University, Stanford CA 943052115, USA.

\section{Abstract}

We examined the elemental (total nitrogen, total organic carbon, and $\mathrm{C} / \mathrm{N})$, isotopic $\left(\delta^{13} \mathrm{C}_{\text {org }}\right.$ and $\left.\delta^{15} \mathrm{~N}_{\text {org }}\right)$, and Rock-Eval pyrolysis $\left(\mathrm{S}_{2}, \mathrm{~S}_{3}\right.$, hydrogen index, oxygen index, and $\left.T_{\max }\right)$ compositions of bulk marine sediments from Hole U1357A in the Adélie Basin, Antarctica, to assess the organic geochemical characteristics and vertical variations in the late Holocene diatomaceous-dominated sediments. Pyrolysis parameters indicate that most of the organic materials are immature and in the Type II stage, indicative of a marine source. In contrast, the results from a conventional crossplot of $\delta^{13} \mathrm{C}_{\text {org }}$ vs. total organic carbon/total nitrogen indicate organic materials that originated as freshwater organic carbon, not marine organic carbon. Given that ${ }^{13} \mathrm{C}$-depleted phytoplankton are dominant in diatomaceous ooze-rich sediments of the Adélie Basin, the apparent dominance of freshwater organic matter is likely attributable to the extremely low $\delta^{13} \mathrm{C}_{\text {org }}(\%)$ values in the bulk sediments.

\section{Introduction}

Operations in Expedition 318 Hole U1357A in the Adélie Basin, located on the Antarctic continental shelf off the Wilkes Land margin, led to the recovery of a long core of Holocene diatomaceous ooze-dominated sediment. The aim of this expedition was to develop the first annually resolved time series of Holocene oceanographic and climatic variability (see the "Expedition 318 summary" chapter [Expedition 318 Scientists, 2011]). Reconstruction of coastal environmental change in the Holocene has traditionally been achieved using a combination of biological and physical indicators (e.g., pollen, diatoms, foraminifers, and sediment grain size). In addition to these traditional indicators in Holocene sediments, organic geochemical proxies such as total organic carbon (TOC), $\mathrm{C} / \mathrm{N}, \delta^{13} \mathrm{C}_{\text {org }}$, and $\delta^{15} \mathrm{~N}_{\text {org }}$ have also been used to reconstruct oceanographic processes and to understand the origin of the organic materials in these sediments (Lamb et al., 2006; Jacot Des Combes et al., 2008). Rock-Eval pyrolysis is the most basic organic geochemical analysis of sedimentary organic matter, providing valuable parameters, including $S_{1}, S_{2}$, and $S_{3}$ peaks, hydrogen index (HI), oxygen index (OI), and $T_{\max }$ to aid discrimination of the type and maturity of the organic matter. Here, we report several results from organic geochemical analyses 
of the diatomaceous ooze sampled from the Adélie Basin that are vital for understanding the characteristics of the organic matter and identifying the decadal changes in surface productivity during the late Holocene.

\section{Methods}

A total of 276 geochemical samples were collected from Hole U1357A at $20 \mathrm{~cm}$ intervals. The depth of the sampled cores ranges from 0 to $54.35 \mathrm{~m}$. After being freeze-dried for $48 \mathrm{~h}$, all samples were powdered and homogenized using a ball mill. Rock-Eval pyrolysis was performed on powdered bulk samples using the Rock-Eval VI instrument to obtain several valuable indicators, including $\mathrm{S}_{1}, \mathrm{~S}_{2}, \mathrm{HI}, \mathrm{OI}$, and $T_{\max }$. The Rock-Eval pyrolysis method consists of a programmed heating cycle of a small sample $(\sim 100 \mathrm{mg})$ in an inert atmosphere (helium). The pyrolysis oven temperature program was as follows. For $3 \mathrm{~min}$, the oven is kept isothermally at $300^{\circ} \mathrm{C}$ and the free hydrocarbons are volatilized and measured as the $S_{1}$ peak (detected by flame ionization detector [FID]). The temperature is then increased from $300^{\circ}$ to $550^{\circ} \mathrm{C}$ (at $25^{\circ} \mathrm{C} / \mathrm{min}$ ). This is the phase of volatilization of very heavy hydrocarbons compounds $\left(>\mathrm{C}_{40}\right)$ and cracking of nonvolatile organic matter. The hydrocarbons released from this thermal cracking are measured as the $S_{2}$ peak (by FID). The temperature at which $S_{2}$ reaches its maximum depends on the nature and maturity of the kerogen and is called $T_{\max }$. The $\mathrm{CO}_{2}$ emitted from the cracking of kerogen is trapped in the temperature range $300^{\circ}-390^{\circ} \mathrm{C}$. The trap is heated, and $\mathrm{CO}_{2}$ is released and detected by a thermal conductivity detector (TCD) during the cooling of the pyrolysis oven, giving the $S_{3}$ peak (Pimmel and Claypool, 2001). When these components are normalized to the TOC content, the $S_{2}$ peak gives the HI $\left(\mathrm{S}_{2} \times 100 / \mathrm{TOC}\right)$ and $\mathrm{S}_{3}$ gives the OI $\left(S_{3} \times 100 /\right.$ TOC) (Tissot and Welte, 1984; Peters, 1986).

Residual powdered bulk samples were subsampled to make carbonate-free samples. They were treated with $3 \mathrm{~N} \mathrm{HCl}$, diluted to $\mathrm{pH} \mathrm{7,} \mathrm{dried,} \mathrm{and} \mathrm{powdered} \mathrm{again}$ to remove carbonates. TOC, total nitrogen (TN), and stable isotopes of carbon $\left(\delta^{13} \mathrm{C}_{\text {org }}\right)$ and nitrogen $\left(\delta^{15} \mathrm{~N}_{\text {org }}\right)$ in the organic matter were determined using a continuous-flow stable isotope ratio mass spectrometer (IRMS; IsoPrime-EA, Micromass, UK) linked with a CN analyzer (NA Series 2, CE Instruments, Italy) at Seoul National University (Korea). Carbon and nitrogen isotopic compositions $\left(\delta^{13} \mathrm{C}_{\text {org }}\right.$ and $\delta^{15} \mathrm{~N}_{\text {org }}$ ) were calculated using the relation

$$
\delta^{13} \mathrm{C}_{\text {org }} \text { or } \delta^{15} \mathrm{~N}_{\text {org }}(\% 0)=\left[\left(R_{\text {sample }} / R_{\text {standard }}\right)-1\right] \times 1000 \text {, }
$$

where $\mathrm{R}$ is the ratio of ${ }^{13} \mathrm{C} /{ }^{12} \mathrm{C}$ or ${ }^{15} \mathrm{~N} /{ }^{14} \mathrm{~N}$, with a Peedee belemnite (PDB) standard for carbon and an atmospheric $\mathrm{N}_{2}$ standard for nitrogen. Precision of $\delta^{13} \mathrm{C}_{\text {org }}$ and $\delta^{15} \mathrm{~N}_{\text {org }}$ measurements was checked against reference materials procured from the International Atomic Energy Agency. For $\delta^{13} \mathrm{C}_{\text {org }}$ (RM 8542: Glucose ANU, $-10.47 \%$ $\pm 0.13 \%$ ) and $\delta^{15} \mathrm{~N}_{\text {org }}$ (RM 8548: IAEA-N2 +20.3\%o $\pm 0.2 \%$ ), uncertainties in the measurements were less than $0.1 \%$ and $0.2 \%$, respectively.

\section{Results}

The TOC content measured from the Rock-Eval pyrolysis ranges from 1.20 to $2.16 \mathrm{wt} \%$ with an average of $1.67 \mathrm{wt} \%$, whereas the TOC content from the IRMS ranges from 1.06 to $1.86 \mathrm{wt} \%$ with an average of $1.44 \mathrm{wt} \%$. The vertical discrepancy in the TOC contents between these two methods is on average $0.22 \mathrm{wt} \%$, yet both TOC values are higher than those reported from other regions in the Antarctic Peninsula (Khim et al., 2002). TOC contents usually depend on sediment grain size in marine environments, with a negative correlation between TOC and mean grain size. The TN content varies between 0.16 and $0.35 \mathrm{wt} \%$, with the lowest values observed at a depth of 20.94 meters below seafloor (mbsf). TN contents vertically covary with TOC contents, except at $17-21$ and $45-50$ mbsf. $\mathrm{C} / \mathrm{N}$ ratios range from 4.70 to 9.36, with an average value of 6.62 (Table T1; Fig. F1). $\mathrm{C} / \mathrm{N}$ ratios $<10$ generally represent the marine environment, whereas values $>15$ indicate a freshwater environment (Stein et al., 1994). Crosta and Shemesh (2002) report that the $\mathrm{C} / \mathrm{N}$ ratio of diatombound organic matter varies between 3 and 11 in the Southern Ocean.

The $\delta^{13} \mathrm{C}_{\text {org }}$ ratio varies from $-28.33 \%$ to $-21.81 \%$, with an average of $-26.23 \%$. Values display an overall constant vertical trend, except for a distinct excursion in the uppermost part of the core. The vertical variation in the $\delta^{13} \mathrm{C}_{\text {org }}$ ratio is similar to that seen in Core JPC17B from the Adélie Drift (average = $26.6 \%$; Costa et al., 2007). The $\delta^{13} \mathrm{C}_{\text {org }}$ value is also used as a common proxy for the origin of organic matter. If measured $\delta^{13} \mathrm{C}_{\text {org }}$ values lie in the range $-24 \%$ o to $-27 \%$, they indicate a dominance of terrigenous organic matter in the sediment. In contrast, if the values are less than $-24 \%$, the organic matter is thought to be derived from the marine environment (Ruttenberg and Goñi, 1997). The $\delta^{15} \mathrm{~N}_{\text {org }}$ ratio ranges from $2.44 \%$ o to $12.37 \%$, exhibiting a gradual upcore 
decrease in value with the lowest value occurring at the core top (Table T1; Fig. F1). In general, the $\delta^{15} \mathrm{~N}_{\text {org }}$ ratio of terrigenous organic matter ranges widely from $-5 \%$ to $18 \%$, with an average of $3 \%$, and the $\mathrm{C} / \mathrm{N}$ ratio of marine organic matter varies from 7 to 10 (Peters et al., 1978; Schoeninger and DeNiro, $1984)$. In addition, a vertical change in $\delta^{15} \mathrm{~N}_{\text {org }}$ is related to consumption of nutrient nitrate (Jacot Des Combes et al., 2008).

Of the pyrolysis parameters measured during this analysis, $\mathrm{S}_{2}$ values varies between 2.75 and $6.98 \mathrm{mg}$ hydrocarbon (HC)/g rock, with an average of $4.53 \mathrm{mg}$ $\mathrm{HC} / \mathrm{g}$ rock, whereas $\mathrm{S}_{3}$ values range from 1.48 to 4.99 $\mathrm{mg} \mathrm{CO}_{2} / \mathrm{g}$ rock, with an average of $2.43 \mathrm{mg} \mathrm{CO} / \mathrm{g}$ rock. $\mathrm{S}_{2}$ values increase slightly upcore. $\mathrm{HI}$ and OI values are $232-440 \mathrm{mg} \mathrm{HC} / \mathrm{g}$ TOC and 100-299 mg $\mathrm{CO}_{2}$ /TOC, respectively. The vertical variation of $\mathrm{HI}$ is negatively correlated with the OI along the entire sample depth. High HI values are dominant shallower than 12.34 mbsf after an abrupt decrease in values between 16.37 and 12.34 mbsf (Table T1; Fig. F1). A HI value of $<100 \mathrm{mg} \mathrm{HC} / \mathrm{g}$ TOC in marine sediment implies that the TOC is mainly made up of terrigenous organic matter, whereas a $\mathrm{HI}$ value between 200 and $400 \mathrm{mg} \mathrm{HC/g}$ TOC indicates marine organic matter (Tissot and Welte, 1984). $T_{\max }$ values were observed to be between $384^{\circ}$ and $429^{\circ} \mathrm{C}$ along the entire sampled depth. $T_{\max }$ provides an estimate of the organic matter thermal maturity, with values $<435^{\circ} \mathrm{C}$ indicative of immaturity, relative to that of the petroleum generation process (Espitalié et al., 1977; Peters, 1986). Thus, the measured values indicate that most of the organic matter is thermally immature (Table T1).

Correlations of the pyrolysis parameters (HI, OI, $\mathrm{S}_{2}$, and TOC) measured from the Rock-Eval pyrolysis give useful information that can help to elucidate the origin of the organic matter (Type I, Type II, or Type III) through a modified van Krevelen diagram (HI vs. OI) and cross-plot ( $\mathrm{S}_{2}$ vs. TOC). Here, Type I is indicative of organic matter with a lacustrine origin, Type II represents the dominance of marine organic matter, and Type III indicates organic matter with a terrigenous origin (Espitalié et al., 1985). As shown in the modified van Krevelen diagram (Fig. F2A), most of the 276 sample points plot in the Type II area, except for some excursions to Type I, implying that the organic matter primarily originated from a marine source, likely attributed to the surface primary productivity.

Cross-plots of $S_{2}$ vs. TOC show that $S_{2}$ values are strongly correlated with TOC values $\left(\mathrm{r}^{2}>0.98\right)$, having an inclination of 3.13 (Fig. F2B). This means that the organic matter contains $>30 \%$ hydrocarbon that can undergo pyrolysis. It also shows that the high organic carbon content, ranging from 1 to $2 \mathrm{wt} \%$, belongs to Type II, as shown in the van Krevelen diagram.

In the coastal environment where organic matter sources are mixed, typical ranges of $\delta^{13} \mathrm{C}_{\text {org }}$ values and the $\mathrm{C} / \mathrm{N}$ ratios can be used to classify the sources of organic matter. Terrestrial plants (C3) using the Calvin photosynthetic pathway have $\delta^{13} \mathrm{C}_{\text {org }}$ values between $-32 \%$ and $-21 \%$, whereas C4 plants utilizing the Hatch-Slack photosynthetic pathway have $\delta^{13} \mathrm{C}_{\text {org }}$ values ranging from $-17 \%$ o to $-9 \%$ (Deines, 1980). Terrestrial vegetation normally has $C / N$ ratios $>12$ (Prahl et al., 1980). The $\delta^{13} \mathrm{C}_{\text {org }}$ and $\mathrm{C} / \mathrm{N}$ ratios for marine particulate organic carbon (POC) are limited to $-24 \%$ o to $-18 \%$ and 5 to 10 , respectively, whereas the $\delta^{13} \mathrm{C}_{\text {org }}$ and $\mathrm{C} / \mathrm{N}$ ratios for freshwater POC vary from $-33 \%$ to $-25 \%$ and 4 to 10 , respectively (Lamb et al., 2006).

As shown in Figure F3, most of the data points measured from Hole U1357A were classified as freshwater algae. The results seem to indicate that the organic matter is strongly affected by freshwater algae rather than marine algae. However, the extremely low $\delta^{13} \mathrm{C}_{\text {org }}$ values observed here are related to a general ${ }^{13} \mathrm{C}$ depletion in phytoplankton in Southern Ocean surface waters. It is also known that $\delta^{13} \mathrm{C}_{\text {org }}$ values across the Antarctic and Southern Ocean can range from $-25 \%$ o to $30 \%$ (Gibson et al., 1999).

\section{Acknowledgments}

This study used samples provided by the Integrated Ocean Drilling Program (IODP) and was supported by the study on Korea Integrated Ocean Drilling Program of the Korea Institute of Geoscience and Mineral Resources (KIGAM) funded by the Ministry of Land, Transport and Maritime Affairs of Korea.

\section{References}

Costa, E., Dunbar, R.B., Kryc, K.A., Mucciarone, D.A., Brachfeld, S., Roark, E.B., Manley, P.L., Murray, R.W., and Leventer, A., 2007. Solar forcing and El NiñoSouthern Oscillation (ENSO) influences on productivity cycles interpreted from a late Holocene high-resolution marine sediment record, Adélie Drift, East Antarctic margin. In Cooper, A.K., and Raymond, C.R., et al. (Eds.), Antarctica: A Keystone in a Changing World. USGS Open-File Rep., 2007-1047:1-6. doi:10.3133/of20071047.srp036

Crosta, X., and Shemesh, A., 2002. Reconciling down core anticorrelation of diatom carbon and nitrogen isotopic 
ratios from the Southern Ocean. Paleoceanography, 17(1):10-1-10-8. doi:10.1029/2000PA000565

Deines, P., 1980. The isotopic composition of reduced organic carbon. In Fritz, P., and Fontes, J.C. (Eds.), Handbook of Environmental Isotope Geochemistry (Vol. 1): The Terrestrial Environment, A: Amsterdam (Elsevier), 329406. doi:10.1016/B978-0-444-41780-0.50015-8

Espitalié, J., Deroo, G., and Marquis, F., 1985. Rock Eval Pyrolysis and Its Applications: Rueil Malmaison (Institut Francais du Petrole).

Espitalié, J., Laporte, J.L., Madec, M., Marquis, F., Leplat, P., Paulet, J., and Boutefeu, A., 1977. Méthode rapide de caractérisation des roches mètres, de leur potentiel pétrolier et de leur degré d'évolution. Rev. Inst. Fr. Pet., 32(1):23-42. doi:10.2516/ogst:1977002

Expedition 318 Scientists, 2011. Expedition 318 summary. In Escutia, C., Brinkhuis, H., Klaus, A., and the Expedition 318 Scientists, Proc. IODP, 318: Tokyo (Integrated Ocean Drilling Program Management International, Inc.). doi:10.2204/iodp.proc.318.101.2011

Gibson, J.A.E., Trull, T., Nichols, P.D., Summons, R.E., and McMinn, A., 1999. Sedimentation of ${ }^{13} \mathrm{C}$-rich organic matter from Antarctic sea-ice algae: a potential indicator of past sea-ice extent. Geology, 27(4):331-334. doi:10.1130/0091-7613(1999)027<0331:SOC$\mathrm{ROM}>2.3 . \mathrm{CO} ; 2$

Jacot Des Combes, H., Esper, O., De La Rocha, C.L., Abelmann, A., Gersonde, R., Yam, R., and Shemesh, A., 2008. Diatom $\delta^{13} \mathrm{C}, \delta^{15} \mathrm{~N}$, and $\mathrm{C} / \mathrm{N}$ since the Last Glacial Maximum in the Southern Ocean: potential impact of species composition. Paleoceanography, 23(4):PA4209. doi:10.1029/2008PA001589

Khim, B.-K., Yoon, H.I., Kang, C.Y., and Bahk, J.J., 2002. Unstable climate oscillations during the late Holocene in the eastern Bransfield Basin, Antarctic peninsula. Quat. Res., 58(3):234-245. doi:10.1006/qres.2002.2371

Lamb, A.L., Wilson, P.G., and Leng, M.J., 2006. A review of coastal palaeoclimate and relative sea-level reconstructions using $\delta^{13} \mathrm{C}$ and $\mathrm{C} / \mathrm{N}$ ratios in organic material. Earth-Sci. Rev., 75(1-4):29-57 doi:10.1016/j.earscirev.2005.10.003
Peters, K.E., 1986. Guidelines for evaluating petroleum source rock using programmed pyrolysis. AAPG Bull., 70(3):318-329. http://archives.datapages.com/data/ bulletns/1986-87/data/pg/0070/0003/0300/ 0318.htm

Peters, K.E., Sweeney, R.E., and Kaplan, I.R., 1978. Correlation of carbon and nitrogen stable isotope ratios in sedimentary organic matter. Limnol. Oceanogr., 23(4):598604. doi:10.4319/1o.1978.23.4.0598

Pimmel, A., and Claypool, G., 2001. Introduction to shipboard organic geochemistry on the JOIDES Resolution. ODP Tech. Note, 30. doi:10.2973/odp.tn.30.2001

Prahl, F.G., Bennett, J.T., and Carpenter, R., 1980. The early diagenesis of aliphatic hydrocarbons and organic matter in sedimentary particulates from Dabob Bay, Washington. Geochim. Cosmochim. Acta, 44(12):1967-1976. doi:10.1016/0016-7037(80)90196-9

Ruttenberg, K.C., and Goñi, M.A., 1997. Phosphorus distribution, $\mathrm{C}: \mathrm{N}: \mathrm{P}$ ratios, and $\delta^{13} \mathrm{C}_{\mathrm{oc}}$ in arctic, temperate, and tropical coastal sediments: tools for characterizing bulk sedimentary organic matter. Mar. Geol., 139(1-4):123145. doi:10.1016/S0025-3227(96)00107-7

Schoeninger, M.J., and DeNiro, M.J., 1984. Nitrogen and carbon isotope composition of bone collagen from marine and terrestrial animals. Geochim. Cosmochim. Acta, 48(4):625-639. doi:10.1016/00167037(84)90091-7

Stein, R., Schubert, C., Vogt, C., and Fütterer, D., 1994. Stable isotope stratigraphy, sedimentation rates, and salinity changes in the latest Pleistocene to Holocene eastern central Arctic Ocean. Mar. Geol., 119(3-4):333-355. doi:10.1016/0025-3227(94)90189-9

Tissot, B.P., and Welte, D.H., 1984. Petroleum Formation and Occurrence (2nd ed.): Heidelberg (Springer-Verlag).

Initial receipt: 28 February 2013

Acceptance: 24 July 2014

Publication: 14 October 2014

MS 318-201 
Figure F1. Variations in the Rock-Eval pyrolysis, elemental analysis, and stable isotopes plotted against core depth, Hole U1357 A. $\mathrm{R}=$ rock, $\mathrm{HI}=$ hydrogen index, TOC = total organic carbon, $\mathrm{OI}=$ oxygen index, $\mathrm{TC}=$ total carbon, $\mathrm{TN}=$ total nitrogen.
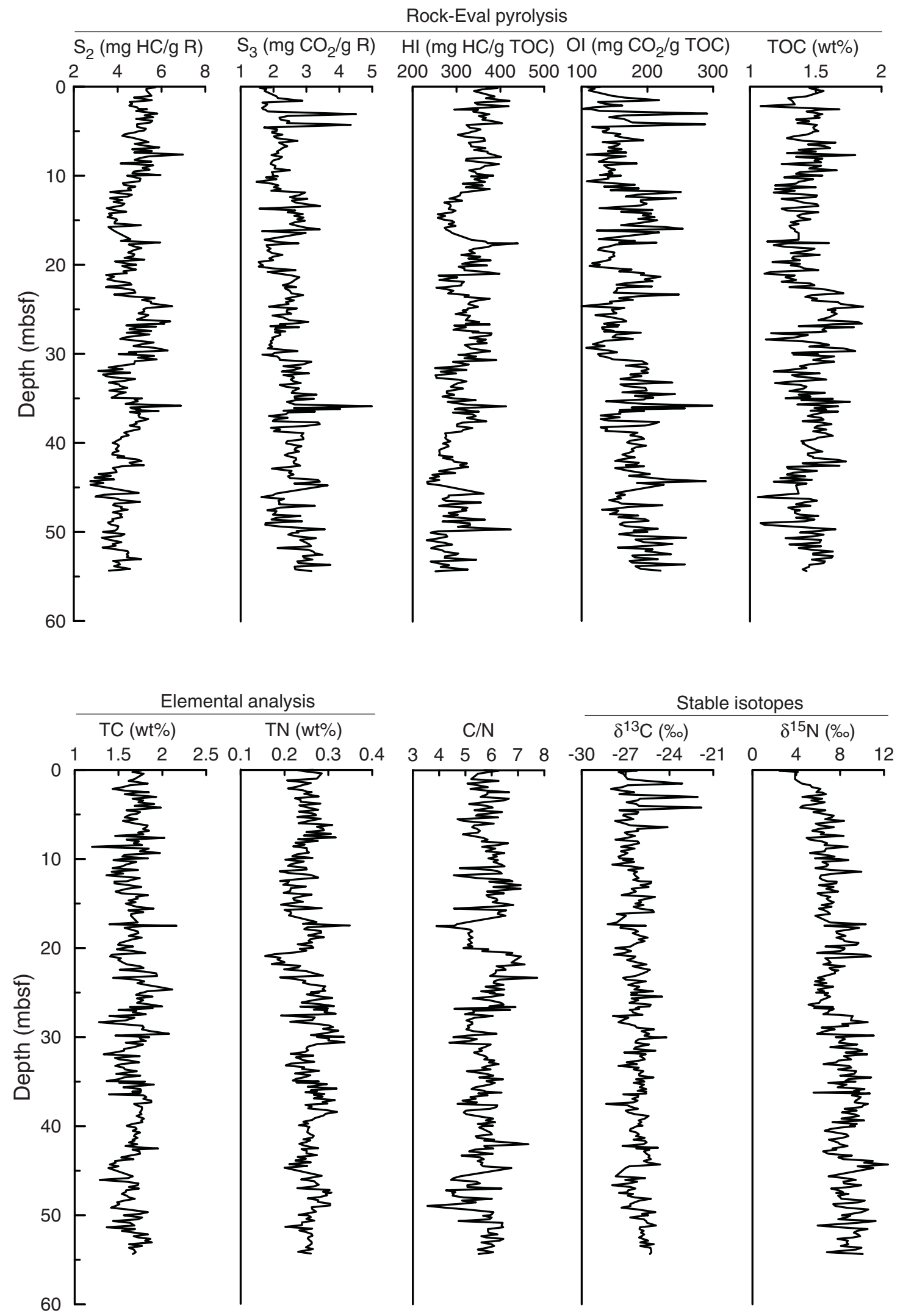
Figure F2. Pyrolysis parameters, Hole U1357A. A. Modified van Krevelen-type diagram. B. Cross-plot for $\mathrm{S}_{2}$ vs. TOC. $\mathrm{HI}=$ hydrogen index, $\mathrm{TOC}=$ total organic carbon, $\mathrm{R}=$ rock.

A

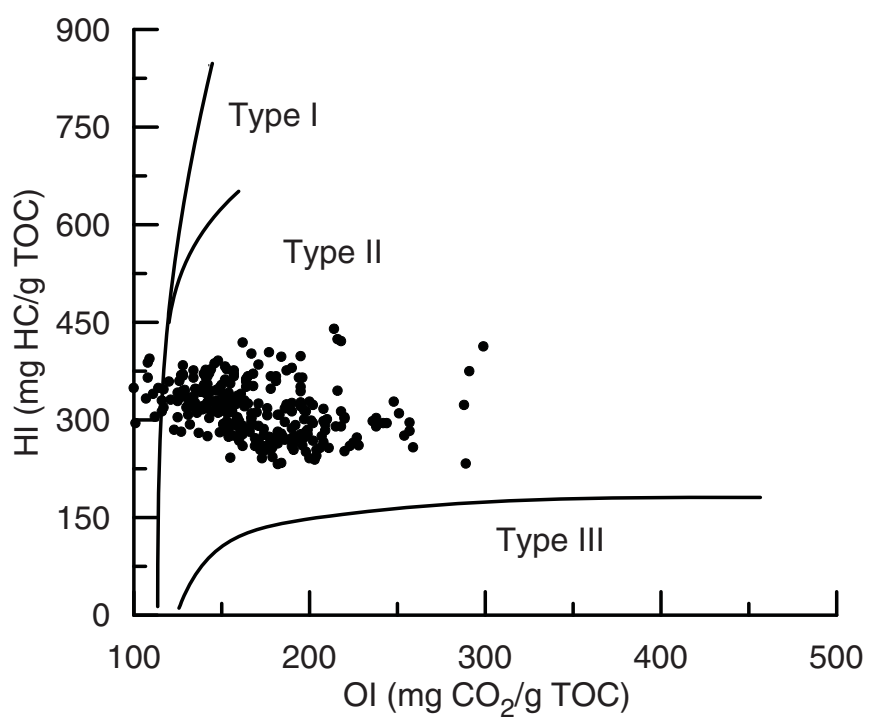

B

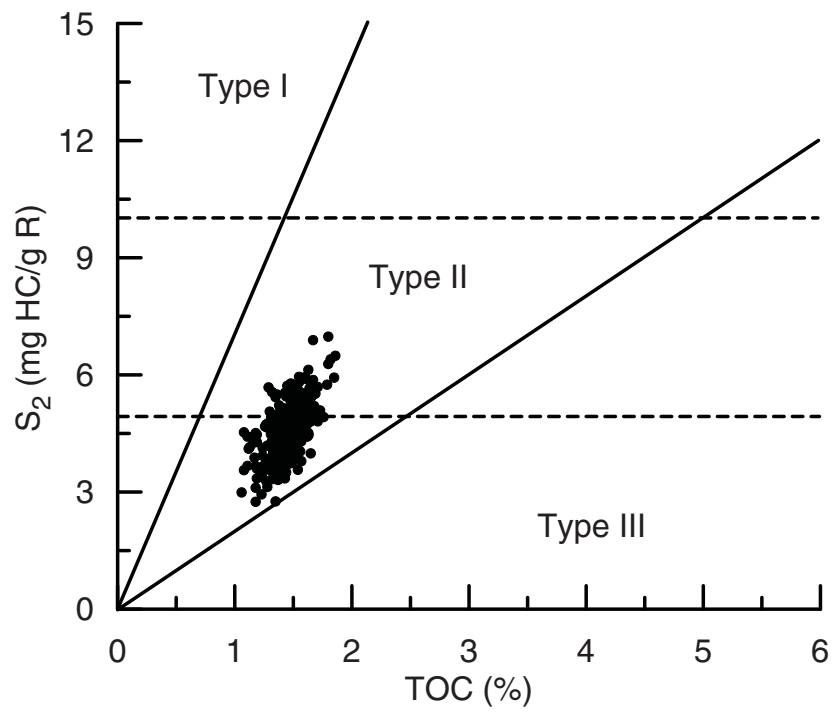


Figure F3. Correlation between $\delta^{13} \mathrm{C}_{\text {org }}$ and $\mathrm{C} / \mathrm{N}$ ratios (after Lamb et al., 2006). POC = particulate organic carbon.

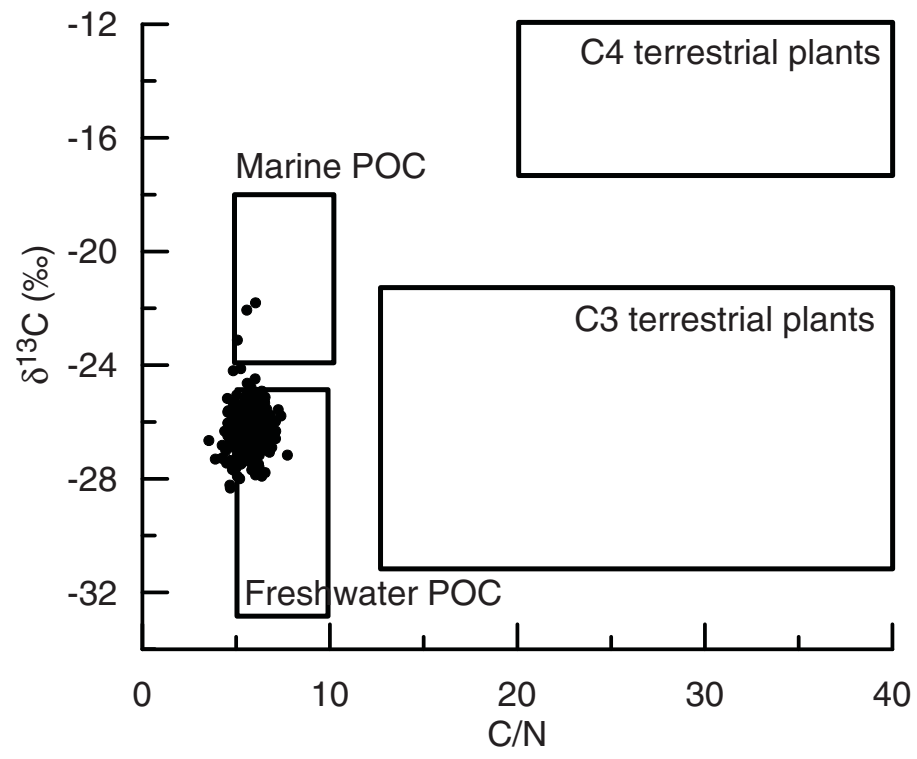


Table T1. Organic geochemical compositions of bulk sediments, Hole U1357A. (Continued on next four pages.)

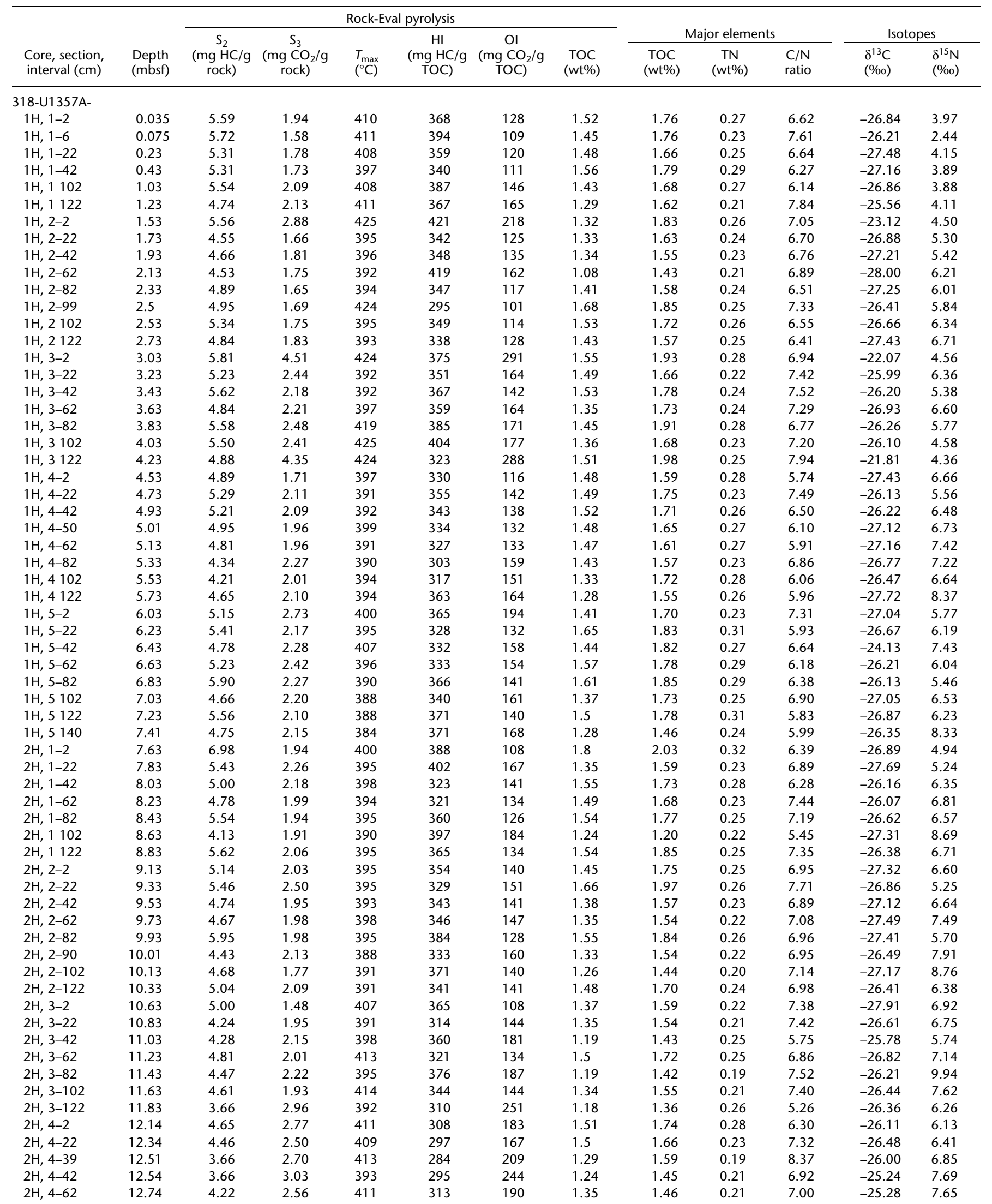


Table T1 continued. (Continued on next page.)

\begin{tabular}{|c|c|c|c|c|c|c|c|c|c|c|c|c|}
\hline \multirow[b]{3}{*}{$\begin{array}{l}\text { Core, section, } \\
\text { interval }(\mathrm{cm})\end{array}$} & \multirow[b]{3}{*}{$\begin{array}{l}\text { Depth } \\
\text { (mbsf) }\end{array}$} & \multicolumn{6}{|c|}{ Rock-Eval pyrolysis } & \multirow{2}{*}{\multicolumn{3}{|c|}{ Major elements }} & \multirow{2}{*}{\multicolumn{2}{|c|}{ Isotopes }} \\
\hline & & \multirow{2}{*}{$\underset{\substack{\mathrm{S}_{2} \\
\text { rock) }}}{\mathrm{mgC} / \mathrm{g}}$} & \multirow{2}{*}{$\underset{\text { rock) }}{\mathrm{S}_{3}}$} & \multirow[b]{2}{*}{$\begin{array}{l}T_{\max } \\
\left({ }^{\circ} \mathrm{C}\right)\end{array}$} & & Ol & & & & & & \\
\hline & & & & & $\underset{\text { TOC) }}{(\mathrm{mg} \mathrm{HC} / \mathrm{g}}$ & $\begin{array}{c}\left(\mathrm{mg} \mathrm{CO}_{2} / \mathrm{g}\right. \\
\mathrm{TOC})\end{array}$ & $\begin{array}{l}\text { TOC } \\
\text { (wt\%) }\end{array}$ & $\begin{array}{l}\text { TOC } \\
\text { (wt\%) }\end{array}$ & $\begin{array}{c}\mathrm{TN} \\
(w t \%)\end{array}$ & $\begin{array}{l}\mathrm{C} / \mathrm{N} \\
\text { ratio }\end{array}$ & $\begin{array}{l}\delta^{13} \mathrm{C} \\
(\% o)\end{array}$ & $\begin{array}{l}\delta^{15} \mathrm{~N} \\
(\% o)\end{array}$ \\
\hline $2 \mathrm{H}, 4-82$ & 12.94 & 3.81 & 2.66 & 420 & 272 & 190 & 1.4 & 1.62 & 0.20 & 8.23 & -25.98 & 6.07 \\
\hline $2 \mathrm{H}, 4-102$ & 13.14 & 4.31 & 3.01 & 395 & 285 & 199 & 1.51 & 1.76 & 0.25 & 7.07 & -25.42 & 7.40 \\
\hline $2 \mathrm{H}, 4-122$ & 13.34 & 4.21 & 3.42 & 411 & 287 & 190 & 1.52 & 1.60 & 0.21 & 7.47 & -26.59 & 6.74 \\
\hline $2 \mathrm{H}, 5-2$ & 13.65 & 3.50 & 1.57 & 425 & 282 & 127 & 1.24 & 1.47 & 0.21 & 6.89 & -26.29 & 7.83 \\
\hline $2 \mathrm{H}, 5-22$ & 13.85 & 3.81 & 2.66 & 407 & 298 & 208 & 1.28 & 1.53 & 0.20 & 7.74 & -26.67 & 5.94 \\
\hline $2 \mathrm{H}, 5-42$ & 14.05 & 4.39 & 2.43 & 414 & 289 & 160 & 1.52 & 1.84 & 0.26 & 7.01 & -27.27 & 6.28 \\
\hline $2 \mathrm{H}, 5-62$ & 14.25 & 3.60 & 2.89 & 423 & 257 & 206 & 1.4 & 1.70 & 0.24 & 7.14 & -24.99 & 7.11 \\
\hline $2 \mathrm{H}, 5-82$ & 14.45 & 3.73 & 2.63 & 419 & 274 & 193 & 1.36 & 1.72 & 0.22 & 7.82 & -25.50 & 6.77 \\
\hline $2 \mathrm{H}, 5-102$ & 14.65 & 3.54 & 2.91 & 406 & 257 & 211 & 1.38 & 1.62 & 0.22 & 7.27 & -26.09 & 6.95 \\
\hline $2 \mathrm{H}, 5-122$ & 14.85 & 3.94 & 2.72 & 419 & 270 & 186 & 1.46 & 1.70 & 0.24 & 7.01 & -25.94 & 7.42 \\
\hline $2 \mathrm{H}, 5-138$ & 15.01 & 3.97 & 2.95 & 410 & 290 & 215 & 1.37 & 1.58 & 0.21 & 7.43 & -26.33 & 7.20 \\
\hline $2 \mathrm{H}, 6-2$ & 15.17 & 3.87 & 2.65 & 419 & 295 & 202 & 1.31 & 1.64 & 0.19 & 8.54 & -25.77 & 6.32 \\
\hline $2 \mathrm{H}, 6-22$ & 15.37 & 3.86 & 2.50 & 413 & 282 & 182 & 1.37 & 1.71 & 0.23 & 7.49 & -26.45 & 7.22 \\
\hline $2 \mathrm{H}, 6-42$ & 15.57 & 5.06 & 2.72 & 409 & 298 & 160 & 1.3 & 1.90 & 0.29 & 6.67 & -26.04 & 6.07 \\
\hline $2 \mathrm{H}, 6-62$ & 15.77 & 3.58 & 2.97 & 418 & 273 & 227 & 1.31 & 1.61 & 0.20 & 8.07 & -25.13 & 6.58 \\
\hline $2 \mathrm{H}, 6-82$ & 15.97 & 3.70 & 3.41 & 422 & 276 & 254 & 1.34 & 1.78 & 0.21 & 8.33 & -25.06 & 6.91 \\
\hline $2 \mathrm{H}, 6-102$ & 16.17 & 3.82 & 1.65 & 426 & 285 & 123 & 1.34 & 1.65 & 0.22 & 7.65 & -27.60 & 7.09 \\
\hline $2 \mathrm{H}, 6-122$ & 16.37 & 3.97 & 2.98 & 401 & 290 & 218 & 1.37 & 1.64 & 0.21 & 7.82 & -26.98 & 5.73 \\
\hline $3 \mathrm{H}, 1-2$ & 17.13 & 4.58 & 1.73 & 413 & 334 & 126 & 1.37 & 1.72 & 0.27 & 6.30 & -27.60 & 7.11 \\
\hline $3 \mathrm{H}, 1-22$ & 17.33 & 4.15 & 2.04 & 386 & 367 & 181 & 1.13 & 1.40 & 0.24 & 5.77 & -28.23 & 10.35 \\
\hline $3 \mathrm{H}, 1-40$ & 17.51 & 5.94 & 2.51 & 424 & 371 & 157 & 1.6 & 2.16 & 0.35 & 6.19 & -25.61 & 6.56 \\
\hline $3 \mathrm{H}, 1-42$ & 17.53 & 5.68 & 2.76 & 393 & 440 & 214 & 1.29 & 1.77 & 0.33 & 5.36 & -27.31 & 7.15 \\
\hline $3 \mathrm{H}, 1-62$ & 17.73 & 4.51 & 1.79 & 396 & 382 & 152 & 1.18 & 1.57 & 0.25 & 6.23 & -27.36 & 9.74 \\
\hline $3 \mathrm{H}, 1-82$ & 17.93 & 4.73 & 1.81 & 392 & 372 & 143 & 1.27 & 1.64 & 0.25 & 6.71 & -26.40 & 8.81 \\
\hline $3 \mathrm{H}, 1-102$ & 18.13 & 5.08 & 1.91 & 393 & 341 & 128 & 1.49 & 1.72 & 0.28 & 6.07 & -26.15 & 7.65 \\
\hline $3 \mathrm{H}, 1-122$ & 18.33 & 4.77 & 1.81 & 408 & 329 & 125 & 1.45 & 1.73 & 0.28 & 6.08 & -26.51 & 7.71 \\
\hline $3 \mathrm{H}, 2-2$ & 18.63 & 4.48 & 1.96 & 396 & 342 & 150 & 1.31 & 1.62 & 0.25 & 6.37 & -25.99 & 8.50 \\
\hline $3 \mathrm{H}, 2-22$ & 18.83 & 4.76 & 2.27 & 420 & 311 & 148 & 1.53 & 1.77 & 0.29 & 6.09 & -25.49 & 7.67 \\
\hline $3 \mathrm{H}, 2-42$ & 19.03 & 4.71 & 2.00 & 412 & 354 & 150 & 1.33 & 1.66 & 0.26 & 6.38 & -26.10 & 8.28 \\
\hline $3 \mathrm{H}, 2-62$ & 19.23 & 4.31 & 1.87 & 390 & 327 & 142 & 1.32 & 1.59 & 0.26 & 6.23 & -25.70 & 8.41 \\
\hline $3 \mathrm{H}, 2-82$ & 19.43 & 5.22 & 1.99 & 395 & 378 & 144 & 1.38 & 1.52 & 0.26 & 5.76 & -26.20 & 9.68 \\
\hline $3 \mathrm{H}, 2-102$ & 19.63 & 3.88 & 1.55 & 409 & 332 & 132 & 1.17 & 1.50 & 0.23 & 6.50 & -26.46 & 9.51 \\
\hline $3 \mathrm{H}, 2-122$ & 19.83 & 4.32 & 1.60 & 413 & 313 & 116 & 1.38 & 1.67 & 0.26 & 6.37 & -26.66 & 6.66 \\
\hline $3 \mathrm{H}, 2-140$ & 20.01 & 4.85 & 1.68 & 391 & 370 & 128 & 1.31 & 1.55 & 0.27 & 5.82 & -27.70 & 8.41 \\
\hline $3 \mathrm{H}, 3-2$ & 20.14 & 4.21 & 1.55 & 418 & 305 & 112 & 1.38 & 1.48 & 0.24 & 6.30 & -27.00 & 8.38 \\
\hline $3 \mathrm{H}, 3-22$ & 20.34 & 4.48 & 1.97 & 408 & 318 & 140 & 1.41 & 1.67 & 0.25 & 6.69 & -26.62 & 8.00 \\
\hline $3 \mathrm{H}, 3-42$ & 20.54 & 4.84 & 2.67 & 396 & 318 & 176 & 1.52 & 1.81 & 0.22 & 8.06 & -27.07 & 5.90 \\
\hline $3 \mathrm{H}, 3-62$ & 20.74 & 4.26 & 1.82 & 386 & 367 & 157 & 1.16 & 1.43 & 0.18 & 8.05 & -27.79 & 10.33 \\
\hline $3 \mathrm{H}, 3-82$ & 20.94 & 4.42 & 2.17 & 387 & 398 & 195 & 1.11 & 1.40 & 0.16 & 9.00 & -26.33 & 10.81 \\
\hline $3 \mathrm{H}, 3-102$ & 21.14 & 3.47 & 2.55 & 409 & 259 & 190 & 1.34 & 1.53 & 0.19 & 8.00 & -26.47 & 6.65 \\
\hline $3 \mathrm{H}, 3-122$ & 21.34 & 3.84 & 2.79 & 384 & 302 & 220 & 1.27 & 1.50 & 0.18 & 8.14 & -26.90 & 6.89 \\
\hline $3 \mathrm{H}, 4-2$ & 21.64 & 3.53 & 2.65 & 425 & 261 & 196 & 1.35 & 1.55 & 0.20 & 7.77 & -26.14 & 7.14 \\
\hline $3 \mathrm{H}, 4-22$ & 21.84 & 3.93 & 2.58 & 423 & 317 & 208 & 1.24 & 1.60 & 0.17 & 9.36 & -25.57 & 6.47 \\
\hline $3 \mathrm{H}, 4-42$ & 22.04 & 4.55 & 2.48 & 386 & 312 & 170 & 1.46 & 1.75 & 0.24 & 7.40 & -26.51 & 8.43 \\
\hline $3 \mathrm{H}, 4-62$ & 22.24 & 4.63 & 2.28 & 418 & 311 & 153 & 1.49 & 1.79 & 0.23 & 7.73 & -25.99 & 7.02 \\
\hline $3 \mathrm{H}, 4-82$ & 22.44 & 3.57 & 2.69 & 409 & 275 & 207 & 1.3 & 1.51 & 0.20 & 7.61 & -25.33 & 7.25 \\
\hline $3 \mathrm{H}, 4-89$ & 22.51 & 3.46 & 2.34 & 421 & 254 & 172 & 1.36 & 1.61 & 0.22 & 7.29 & -25.46 & 7.84 \\
\hline $3 \mathrm{H}, 4-102$ & 22.64 & 4.22 & 2.37 & 422 & 274 & 154 & 1.54 & 1.80 & 0.25 & 7.18 & -25.52 & 7.51 \\
\hline $3 \mathrm{H}, 4-122$ & 22.84 & 4.74 & 2.49 & 419 & 293 & 154 & 1.62 & 1.93 & 0.26 & 7.31 & -25.82 & 7.52 \\
\hline $3 \mathrm{H}, 5-2$ & 23.14 & 4.81 & 2.54 & 417 & 281 & 149 & 1.71 & 1.94 & 0.29 & 6.74 & -26.34 & 6.60 \\
\hline $3 \mathrm{H}, 5-22$ & 23.34 & 3.84 & 2.90 & 386 & 328 & 248 & 1.47 & 1.44 & 0.19 & 7.56 & -27.17 & 5.97 \\
\hline $3 \mathrm{H}, 5-42$ & 23.54 & 4.52 & 2.64 & 392 & 318 & 186 & 1.42 & 1.70 & 0.23 & 7.51 & -26.99 & 6.57 \\
\hline $3 \mathrm{H}, 5-62$ & 23.74 & 5.68 & 2.37 & 394 & 376 & 157 & 1.51 & 1.80 & 0.25 & 7.24 & -25.61 & 5.71 \\
\hline $3 \mathrm{H}, 5-82$ & 23.94 & 5.11 & 2.62 & 415 & 348 & 178 & 1.47 & 1.72 & 0.24 & 7.09 & -26.36 & 6.76 \\
\hline $3 \mathrm{H}, 5-102$ & 24.14 & 5.52 & 2.38 & 398 & 331 & 143 & 1.67 & 1.82 & 0.26 & 7.02 & -26.82 & 5.66 \\
\hline $3 \mathrm{H}, 5-122$ & 24.34 & 5.52 & 2.46 & 421 & 327 & 146 & 1.69 & 1.94 & 0.29 & 6.63 & -26.06 & 7.37 \\
\hline $3 \mathrm{H}, 6-2$ & 24.65 & 6.49 & 1.86 & 425 & 349 & 100 & 1.86 & 2.12 & 0.29 & 7.37 & -26.40 & 5.97 \\
\hline $3 \mathrm{H}, 6-22$ & 24.85 & 5.33 & 2.71 & 417 & 325 & 165 & 1.64 & 1.83 & 0.29 & 6.24 & -25.12 & 6.53 \\
\hline $3 \mathrm{H}, 6-38$ & 25.01 & 5.17 & 2.42 & 404 & 325 & 152 & 1.59 & 1.78 & 0.26 & 6.85 & -26.62 & 5.82 \\
\hline $3 \mathrm{H}, 6-42$ & 25.05 & 5.18 & 2.41 & 419 & 314 & 146 & 1.65 & 1.75 & 0.28 & 6.26 & -26.21 & 6.91 \\
\hline $3 \mathrm{H}, 6-62$ & 25.25 & 5.22 & 2.40 & 411 & 322 & 148 & 1.62 & 1.70 & 0.25 & 6.78 & -26.63 & 6.58 \\
\hline $3 \mathrm{H}, 6-82$ & 25.45 & 4.95 & 2.54 & 423 & 298 & 153 & 1.66 & 1.89 & 0.28 & 6.84 & -24.49 & 7.10 \\
\hline $3 \mathrm{H}, 6-102$ & 25.65 & 5.40 & 1.97 & 407 & 331 & 121 & 1.63 & 1.71 & 0.31 & 5.54 & -26.59 & 6.70 \\
\hline $3 \mathrm{H}, 6-122$ & 25.85 & 5.23 & 2.28 & 418 & 327 & 142 & 1.6 & 1.78 & 0.27 & 6.58 & -26.14 & 6.25 \\
\hline $3 \mathrm{H}, 7-2$ & 26.16 & 4.88 & 2.48 & 414 & 321 & 163 & 1.52 & 1.71 & 0.24 & 7.16 & -26.73 & 6.09 \\
\hline $3 \mathrm{H}, 7-22$ & 26.36 & 6.40 & 3.06 & 393 & 352 & 168 & 1.82 & 1.87 & 0.28 & 6.62 & -25.28 & 5.11 \\
\hline $3 \mathrm{H}, 7-42$ & 26.56 & 5.93 & 2.57 & 421 & 321 & 139 & 1.85 & 2.00 & 0.31 & 6.38 & -25.36 & 5.73 \\
\hline
\end{tabular}


Table T1 continued. (Continued on next page.)

\begin{tabular}{|c|c|c|c|c|c|c|c|c|c|c|c|c|}
\hline \multirow[b]{3}{*}{$\begin{array}{l}\text { Core, section, } \\
\text { interval }(\mathrm{cm})\end{array}$} & \multirow[b]{3}{*}{$\begin{array}{l}\text { Depth } \\
\text { (mbsf) }\end{array}$} & \multicolumn{6}{|c|}{ Rock-Eval pyrolysis } & \multirow{2}{*}{\multicolumn{3}{|c|}{ Major elements }} & \multirow{2}{*}{\multicolumn{2}{|c|}{ Isotopes }} \\
\hline & & \multirow{2}{*}{$\underset{\substack{\mathrm{S}_{2} \\
\text { rock) }}}{\mathrm{mgC} / \mathrm{g}}$} & \multirow{2}{*}{$\underset{\text { rock) }}{\mathrm{S}_{3}}$} & \multirow[b]{2}{*}{$\begin{array}{l}T_{\max } \\
\left({ }^{\circ} \mathrm{C}\right)\end{array}$} & & & & & & & & \\
\hline & & & & & $\begin{array}{c}(\mathrm{mg} \mathrm{HC} / \mathrm{g} \\
\mathrm{TOC})\end{array}$ & $\begin{array}{c}\left(\mathrm{mg} \mathrm{CO}_{2} / \mathrm{g}\right. \\
\mathrm{TOC})\end{array}$ & $\begin{array}{c}\text { TOC } \\
\text { (wt\%) }\end{array}$ & $\begin{array}{c}\text { TOC } \\
(w t \%)\end{array}$ & $\begin{array}{c}\mathrm{TN} \\
(\mathrm{wt} \%)\end{array}$ & $\begin{array}{l}\mathrm{C} / \mathrm{N} \\
\text { ratio }\end{array}$ & $\begin{array}{l}\delta^{13} \mathrm{C} \\
(\% o)\end{array}$ & $\begin{array}{l}\delta^{15} \mathrm{~N} \\
(\% \mathrm{o})\end{array}$ \\
\hline $3 \mathrm{H}, 7-62$ & 26.63 & 6.13 & 2.19 & 406 & 376 & 134 & 1.63 & 1.63 & 0.24 & 6.91 & -26.13 & 7.35 \\
\hline $3 \mathrm{H}, 7-82$ & 26.76 & 4.43 & 2.36 & 420 & 295 & 157 & 1.5 & 1.89 & 0.29 & 6.52 & -26.30 & 5.63 \\
\hline $4 \mathrm{H}, 1-2$ & 26.83 & 4.39 & 1.90 & 409 & 320 & 139 & 1.37 & 1.74 & 0.30 & 5.82 & -26.06 & 7.42 \\
\hline $4 \mathrm{H}, 1-22$ & 26.96 & 5.75 & 2.78 & 393 & 321 & 155 & 1.79 & 1.50 & 0.27 & 5.62 & -26.72 & 7.28 \\
\hline $4 \mathrm{H}, 1-42$ & 27.03 & 4.86 & 2.07 & 405 & 308 & 131 & 1.58 & 1.70 & 0.30 & 5.72 & -26.55 & 6.98 \\
\hline $4 \mathrm{H}, 1-62$ & 27.23 & 4.54 & 2.04 & 409 & 293 & 132 & 1.55 & 1.66 & 0.29 & 5.72 & -26.50 & 6.91 \\
\hline $4 \mathrm{H}, 1-82$ & 27.43 & 5.53 & 2.36 & 411 & 350 & 149 & 1.58 & 1.81 & 0.32 & 5.72 & -26.10 & 6.47 \\
\hline $4 \mathrm{H}, 1-90$ & 27.51 & 5.11 & 2.04 & 416 & 336 & 134 & 1.52 & 1.75 & 0.28 & 6.30 & -25.84 & 7.91 \\
\hline $4 \mathrm{H}, 1-102$ & 27.63 & 4.41 & 2.20 & 390 & 380 & 190 & 1.16 & 1.40 & 0.19 & 7.27 & -27.87 & 9.16 \\
\hline $4 \mathrm{H}, 1-122$ & 27.83 & 5.45 & 2.21 & 387 & 378 & 153 & 1.44 & 1.68 & 0.27 & 6.25 & -26.81 & 9.05 \\
\hline $4 \mathrm{H}, 2-2$ & 28.13 & 4.39 & 1.96 & 396 & 330 & 147 & 1.33 & 1.58 & 0.26 & 5.98 & -27.24 & 8.99 \\
\hline $4 \mathrm{H}, 2-22$ & 28.33 & 4.11 & 1.99 & 388 & 367 & 178 & 1.12 & 1.28 & 0.21 & 6.00 & -27.49 & 10.52 \\
\hline $4 \mathrm{H}, 2-42$ & 28.53 & 4.86 & 1.91 & 407 & 347 & 136 & 1.4 & 1.53 & 0.27 & 5.75 & -26.80 & 8.19 \\
\hline $4 \mathrm{H}, 2-62$ & 28.73 & 5.64 & 1.95 & 393 & 369 & 127 & 1.53 & 1.78 & 0.30 & 5.90 & -26.35 & 8.76 \\
\hline $4 \mathrm{H}, 2-82$ & 28.93 & 5.10 & 1.87 & 423 & 319 & 117 & 1.6 & 1.79 & 0.31 & 5.75 & -26.24 & 6.31 \\
\hline $4 \mathrm{H}, 2-102$ & 29.13 & 4.72 & 1.96 & 421 & 326 & 135 & 1.45 & 1.77 & 0.29 & 6.14 & -25.05 & 7.51 \\
\hline $4 \mathrm{H}, 2-122$ & 29.33 & 5.69 & 1.83 & 413 & 333 & 107 & 1.71 & 1.86 & 0.32 & 5.76 & -25.90 & 6.63 \\
\hline $4 \mathrm{H}, 3-2$ & 29.63 & 6.28 & 2.73 & 410 & 377 & 141 & 1.8 & 2.08 & 0.29 & 7.14 & -25.39 & 5.90 \\
\hline $4 \mathrm{H}, 3-22$ & 29.83 & 4.51 & 2.03 & 409 & 342 & 154 & 1.32 & 1.46 & 0.26 & 5.63 & -25.71 & 11.08 \\
\hline $4 \mathrm{H}, 3-40$ & 30.01 & 4.85 & 1.97 & 424 & 319 & 130 & 1.52 & 1.85 & 0.34 & 5.53 & -25.18 & 7.60 \\
\hline $4 \mathrm{H}, 3-42$ & 30.03 & 4.04 & 1.66 & 426 & 304 & 125 & 1.33 & 1.74 & 0.27 & 6.36 & -24.20 & 8.10 \\
\hline $4 \mathrm{H}, 3-62$ & 30.23 & 5.66 & 2.10 & 414 & 345 & 128 & 1.64 & 1.75 & 0.30 & 5.79 & -26.07 & 8.44 \\
\hline $4 \mathrm{H}, 3-82$ & 30.43 & 4.93 & 2.18 & 410 & 318 & 141 & 1.55 & 1.81 & 0.30 & 6.04 & -26.28 & 8.01 \\
\hline $4 \mathrm{H}, 3-102$ & 30.63 & 5.78 & 2.19 & 412 & 391 & 148 & 1.48 & 1.58 & 0.34 & 4.70 & -26.33 & 7.90 \\
\hline $4 \mathrm{H}, 3-122$ & 30.83 & 4.79 & 3.15 & 424 & 292 & 192 & 1.64 & 1.78 & 0.28 & 6.48 & -25.39 & 9.57 \\
\hline $4 \mathrm{H}, 4-2$ & 31.13 & 4.75 & 2.90 & 411 & 328 & 200 & 1.45 & 1.68 & 0.25 & 6.63 & -26.30 & 7.11 \\
\hline $4 \mathrm{H}, 4-22$ & 31.33 & 4.18 & 2.30 & 412 & 303 & 167 & 1.38 & 1.57 & 0.25 & 6.26 & -26.43 & 9.46 \\
\hline $4 \mathrm{H}, 4-42$ & 31.53 & 3.59 & 2.83 & 424 & 251 & 198 & 1.43 & 1.56 & 0.25 & 6.16 & -24.96 & 10.03 \\
\hline $4 \mathrm{H}, 4-62$ & 31.73 & 4.23 & 2.64 & 420 & 323 & 202 & 1.31 & 1.45 & 0.25 & 5.91 & -27.10 & 8.50 \\
\hline $4 \mathrm{H}, 4-82$ & 31.93 & 3.11 & 2.25 & 424 & 264 & 191 & 1.18 & 1.33 & 0.21 & 6.22 & -25.62 & 10.48 \\
\hline $4 \mathrm{H}, 4-102$ & 32.13 & 4.58 & 3.08 & 414 & 299 & 201 & 1.53 & 1.74 & 0.27 & 6.55 & -26.02 & 8.36 \\
\hline $4 \mathrm{H}, 4-122$ & 32.33 & 3.33 & 2.30 & 424 & 252 & 174 & 1.32 & 1.46 & 0.23 & 6.46 & -25.80 & 8.70 \\
\hline $4 \mathrm{H}, 4-140$ & 32.51 & 3.46 & 2.60 & 424 & 254 & 191 & 1.36 & 1.51 & 0.24 & 6.35 & -25.68 & 9.01 \\
\hline $4 \mathrm{H}, 5-2$ & 32.63 & 3.73 & 2.62 & 416 & 254 & 178 & 1.47 & 1.63 & 0.24 & 6.75 & -26.04 & 7.85 \\
\hline $4 \mathrm{H}, 5-22$ & 32.83 & 4.80 & 2.52 & 424 & 304 & 159 & 1.58 & 1.74 & 0.27 & 6.48 & -26.92 & 7.65 \\
\hline $4 \mathrm{H}, 5-42$ & 33.03 & 4.33 & 2.61 & 427 & 323 & 195 & 1.34 & 1.53 & 0.21 & 7.17 & -26.22 & 6.49 \\
\hline $4 \mathrm{H}, 5-62$ & 33.23 & 3.60 & 2.83 & 427 & 303 & 238 & 1.19 & 1.46 & 0.20 & 7.21 & -27.49 & 8.82 \\
\hline $4 \mathrm{H}, 5-82$ & 33.43 & 4.06 & 2.19 & 425 & 294 & 159 & 1.38 & 1.56 & 0.25 & 6.37 & -26.10 & 9.02 \\
\hline $4 \mathrm{H}, 5-102$ & 33.63 & 4.11 & 2.37 & 423 & 287 & 166 & 1.43 & 1.57 & 0.24 & 6.55 & -26.44 & 7.82 \\
\hline $4 \mathrm{H}, 5-122$ & 33.83 & 4.44 & 2.78 & 423 & 315 & 197 & 1.41 & 1.71 & 0.28 & 6.13 & -25.78 & 9.47 \\
\hline $4 \mathrm{H}, 6-2$ & 34.13 & 3.62 & 2.59 & 424 & 278 & 199 & 1.3 & 1.48 & 0.22 & 6.58 & -26.08 & 9.29 \\
\hline $4 \mathrm{H}, 6-22$ & 34.33 & 4.37 & 2.55 & 423 & 278 & 162 & 1.57 & 1.75 & 0.26 & 6.75 & -26.06 & 8.01 \\
\hline $4 \mathrm{H}, 6-42$ & 34.53 & 4.04 & 3.31 & 427 & 295 & 242 & 1.37 & 1.56 & 0.25 & 6.30 & -25.09 & 10.84 \\
\hline $4 \mathrm{H}, 6-62$ & 34.73 & 3.91 & 2.64 & 424 & 268 & 181 & 1.46 & 1.50 & 0.23 & 6.61 & -26.28 & 8.73 \\
\hline $4 \mathrm{H}, 6-82$ & 34.93 & 3.72 & 2.65 & 421 & 293 & 209 & 1.27 & 1.37 & 0.22 & 6.23 & -25.76 & 8.48 \\
\hline $4 \mathrm{H}, 6-90$ & 35.01 & 5.11 & 3.23 & 424 & 313 & 198 & 1.63 & 1.80 & 0.28 & 6.48 & -25.72 & 9.40 \\
\hline $4 \mathrm{H}, 6-102$ & 35.13 & 4.78 & 2.71 & 422 & 344 & 195 & 1.39 & 1.51 & 0.23 & 6.65 & -26.45 & 6.92 \\
\hline $4 \mathrm{H}, 6-122$ & 35.33 & 4.92 & 2.41 & 427 & 280 & 137 & 1.76 & 1.90 & 0.30 & 6.41 & -25.74 & 8.59 \\
\hline $4 \mathrm{H}, 7-2$ & 35.63 & 4.36 & 2.65 & 425 & 321 & 195 & 1.36 & 1.56 & 0.25 & 6.15 & -26.03 & 7.78 \\
\hline $4 \mathrm{H}, 7-22$ & 35.83 & 6.89 & 4.99 & 420 & 413 & 299 & 1.67 & 1.75 & 0.32 & 5.49 & -25.59 & 10.23 \\
\hline $4 \mathrm{H}, 7-42$ & 36.03 & 4.58 & 2.62 & 423 & 307 & 176 & 1.49 & 1.76 & 0.26 & 6.77 & -26.18 & 9.28 \\
\hline $4 \mathrm{H}, 7-62$ & 36.13 & 4.64 & 4.03 & 423 & 296 & 257 & 1.57 & 1.66 & 0.27 & 6.05 & -25.58 & 9.74 \\
\hline $4 \mathrm{H}, 7-82$ & 36.23 & 5.03 & 2.96 & 424 & 318 & 187 & 1.58 & 1.75 & 0.25 & 7.06 & -25.65 & 5.57 \\
\hline $5 \mathrm{H}, 1-2$ & 36.33 & 4.85 & 2.30 & 409 & 332 & 158 & 1.46 & 1.72 & 0.27 & 6.48 & -25.58 & 10.72 \\
\hline $5 H, 1-22$ & 36.43 & 5.87 & 3.26 & 409 & 351 & 195 & 1.67 & 1.39 & 0.29 & 4.78 & -27.16 & 9.69 \\
\hline $5 \mathrm{H}, 1-42$ & 36.53 & 4.56 & 2.45 & 422 & 292 & 157 & 1.56 & 1.79 & 0.27 & 6.69 & -26.13 & 9.08 \\
\hline $5 \mathrm{H}, 1-62$ & 36.73 & 5.02 & 2.37 & 417 & 337 & 159 & 1.49 & 1.82 & 0.29 & 6.33 & -26.15 & 9.88 \\
\hline $5 \mathrm{H}, 1-82$ & 36.93 & 4.95 & 1.86 & 412 & 344 & 129 & 1.44 & 1.78 & 0.27 & 6.68 & -26.54 & 9.71 \\
\hline $5 \mathrm{H}, 1-102$ & 37.13 & 4.99 & 2.43 & 422 & 324 & 158 & 1.54 & 1.87 & 0.32 & 5.95 & -26.18 & 9.41 \\
\hline $5 \mathrm{H}, 1-122$ & 37.33 & 5.39 & 2.02 & 424 & 346 & 129 & 1.56 & 1.88 & 0.29 & 6.53 & -26.44 & 9.32 \\
\hline $5 \mathrm{H}, 1-140$ & 37.51 & 5.15 & 1.98 & 415 & 368 & 141 & 1.4 & 1.68 & 0.30 & 5.63 & -28.33 & 10.54 \\
\hline $5 \mathrm{H}, 2-2$ & 37.65 & 4.82 & 3.36 & 425 & 313 & 218 & 1.54 & 1.71 & 0.25 & 6.88 & -26.87 & 10.12 \\
\hline $5 \mathrm{H}, 2-22$ & 37.85 & 4.87 & 3.41 & 426 & 301 & 210 & 1.62 & 1.77 & 0.26 & 6.74 & -26.59 & 9.98 \\
\hline $5 \mathrm{H}, 2-42$ & 38.05 & 4.55 & 2.97 & 426 & 301 & 197 & 1.51 & 1.77 & 0.25 & 6.96 & -26.15 & 8.41 \\
\hline $5 H, 2-62$ & 38.25 & 5.00 & 1.92 & 423 & 336 & 129 & 1.49 & 1.76 & 0.30 & 5.93 & -26.99 & 9.51 \\
\hline $5 \mathrm{H}, 2-82$ & 38.45 & 5.00 & 2.20 & 414 & 316 & 139 & 1.58 & 1.74 & 0.32 & 5.45 & -26.82 & 8.16 \\
\hline $5 \mathrm{H}, 2-102$ & 38.65 & 4.60 & 2.01 & 423 & 311 & 136 & 1.48 & 1.76 & 0.29 & 6.00 & -26.36 & 9.30 \\
\hline
\end{tabular}


Table T1 continued. (Continued on next page.)

\begin{tabular}{|c|c|c|c|c|c|c|c|c|c|c|c|c|}
\hline \multirow[b]{3}{*}{$\begin{array}{l}\text { Core, section, } \\
\text { interval }(\mathrm{cm})\end{array}$} & \multirow[b]{3}{*}{$\begin{array}{l}\text { Depth } \\
\text { (mbsf) }\end{array}$} & \multicolumn{6}{|c|}{ Rock-Eval pyrolysis } & \multirow{2}{*}{\multicolumn{3}{|c|}{ Major elements }} & \multirow{2}{*}{\multicolumn{2}{|c|}{ Isotopes }} \\
\hline & & \multirow{2}{*}{$\underset{\substack{\mathrm{S}_{2} \\
\text { rock) }}}{\mathrm{mg} \mathrm{HC} / \mathrm{g}}$} & \multirow{2}{*}{$\underset{\text { rock) }}{\mathrm{S}_{3}}$} & \multirow[b]{2}{*}{$\begin{array}{l}T_{\max } \\
\left({ }^{\circ} \mathrm{C}\right)\end{array}$} & $\mathrm{HI}$ & Ol & & & & & & \\
\hline & & & & & $\begin{array}{c}(\mathrm{mg} \mathrm{HC} / \mathrm{g} \\
\mathrm{TOC})\end{array}$ & $\begin{array}{c}\left(\mathrm{mg} \mathrm{CO}_{2} / \mathrm{g}\right. \\
\mathrm{TOC})\end{array}$ & $\begin{array}{l}\text { TOC } \\
\text { (wt\%) }\end{array}$ & $\begin{array}{c}\text { TOC } \\
\text { (wt\%) }\end{array}$ & $\begin{array}{c}\mathrm{TN} \\
(\mathrm{wt} \%)\end{array}$ & $\begin{array}{l}\mathrm{C} / \mathrm{N} \\
\text { ratio }\end{array}$ & $\begin{array}{l}\delta^{13} \mathrm{C} \\
(\%)\end{array}$ & $\begin{array}{l}\delta^{15} \mathrm{~N} \\
(\% o)\end{array}$ \\
\hline $5 \mathrm{H}, 2-122$ & 38.85 & 4.30 & 2.91 & 426 & 274 & 185 & 1.57 & 1.71 & 0.29 & 5.91 & -25.42 & 9.72 \\
\hline $5 \mathrm{H}, 3-2$ & 39.15 & 4.51 & 2.84 & 420 & 277 & 174 & 1.63 & 1.79 & 0.27 & 6.66 & -25.69 & 8.12 \\
\hline $5 \mathrm{H}, 3-22$ & 39.35 & 4.17 & 2.90 & 421 & 274 & 191 & 1.52 & 1.76 & 0.26 & 6.69 & -26.10 & 10.20 \\
\hline $5 \mathrm{H}, 3-42$ & 39.55 & 4.14 & 2.91 & 421 & 278 & 195 & 1.49 & 1.77 & 0.24 & 7.30 & -25.92 & 7.30 \\
\hline $5 \mathrm{H}, 3-62$ & 39.75 & 3.97 & 2.42 & 419 & 286 & 174 & 1.39 & 1.66 & 0.25 & 6.53 & -26.13 & 9.52 \\
\hline $5 \mathrm{H}, 3-82$ & 39.95 & 3.84 & 2.38 & 424 & 272 & 169 & 1.41 & 1.59 & 0.23 & 6.84 & -25.84 & 8.90 \\
\hline $5 \mathrm{H}, 3-88$ & 40.01 & 3.77 & 2.39 & 424 & 269 & 171 & 1.4 & 1.61 & 0.25 & 6.48 & -26.24 & 9.37 \\
\hline $5 \mathrm{H}, 3-102$ & 40.15 & 3.97 & 2.59 & 424 & 280 & 182 & 1.42 & 1.69 & 0.26 & 6.59 & -26.43 & 8.05 \\
\hline $5 \mathrm{H}, 4-2$ & 40.32 & 4.06 & 2.85 & 424 & 282 & 198 & 1.44 & 1.71 & 0.25 & 6.72 & -26.67 & 7.13 \\
\hline $5 \mathrm{H}, 4-22$ & 40.52 & 3.99 & 2.67 & 422 & 271 & 182 & 1.47 & 1.66 & 0.25 & 6.57 & -26.70 & 6.60 \\
\hline $5 \mathrm{H}, 4-42$ & 40.72 & 3.91 & 2.64 & 427 & 261 & 176 & 1.5 & 1.75 & 0.25 & 6.93 & -26.25 & 7.60 \\
\hline $5 \mathrm{H}, 4-62$ & 40.92 & 4.04 & 2.78 & 425 & 261 & 179 & 1.55 & 1.69 & 0.26 & 6.55 & -26.09 & 8.59 \\
\hline $5 \mathrm{H}, 4-82$ & 41.12 & 3.77 & 2.47 & 424 & 262 & 172 & 1.44 & 1.68 & 0.27 & 6.28 & -26.29 & 8.76 \\
\hline $5 \mathrm{H}, 4-102$ & 41.32 & 3.75 & 2.34 & 426 & 260 & 162 & 1.44 & 1.72 & 0.26 & 6.50 & -25.70 & 8.20 \\
\hline $5 \mathrm{H}, 4-122$ & 41.52 & 4.30 & 2.53 & 423 & 291 & 171 & 1.48 & 1.66 & 0.24 & 6.84 & -26.46 & 6.97 \\
\hline $5 \mathrm{H}, 5-2$ & 41.63 & 3.98 & 2.68 & 427 & 269 & 181 & 1.48 & 1.68 & 0.26 & 6.50 & -25.68 & 7.27 \\
\hline $5 \mathrm{H}, 5-22$ & 41.83 & 4.83 & 2.69 & 424 & 291 & 162 & 1.66 & 1.62 & 0.25 & 6.48 & -25.56 & 7.81 \\
\hline $5 \mathrm{H}, 5-42$ & 42.03 & 5.09 & 2.61 & 426 & 294 & 151 & 1.73 & 1.71 & 0.23 & 7.32 & -25.79 & 7.99 \\
\hline $5 \mathrm{H}, 5-62$ & 42.23 & 4.54 & 2.62 & 421 & 324 & 187 & 1.4 & 1.58 & 0.25 & 6.45 & -27.21 & 8.68 \\
\hline $5 \mathrm{H}, 5-82$ & 42.43 & 4.72 & 2.78 & 423 & 308 & 182 & 1.53 & 1.80 & 0.26 & 6.81 & -24.78 & 7.73 \\
\hline $5 \mathrm{H}, 5-90$ & 42.51 & 5.20 & 2.81 & 418 & 310 & 167 & 1.68 & 1.95 & 0.28 & 7.05 & -26.27 & 7.30 \\
\hline $5 \mathrm{H}, 5-102$ & 42.63 & 4.19 & 2.50 & 426 & 327 & 195 & 1.28 & 1.66 & 0.24 & 6.95 & -25.37 & 7.67 \\
\hline $5 \mathrm{H}, 5-122$ & 42.83 & 3.78 & 1.95 & 414 & 293 & 151 & 1.29 & 1.74 & 0.25 & 6.92 & -25.74 & 6.45 \\
\hline $5 \mathrm{H}, 6-2$ & 43.1 & 3.93 & 2.55 & 425 & 260 & 169 & 1.51 & 1.75 & 0.25 & 6.98 & -25.57 & 6.94 \\
\hline $5 \mathrm{H}, 6-22$ & 43.3 & 3.95 & 2.47 & 426 & 297 & 186 & 1.33 & 1.63 & 0.27 & 5.95 & -25.35 & 9.08 \\
\hline $5 \mathrm{H}, 6-42$ & 43.5 & 3.13 & 2.61 & 424 & 245 & 204 & 1.28 & 1.53 & 0.23 & 6.66 & -25.44 & 7.84 \\
\hline $5 \mathrm{H}, 6-62$ & 43.7 & 3.69 & 2.44 & 427 & 260 & 172 & 1.42 & 1.67 & 0.25 & 6.71 & -25.27 & 8.30 \\
\hline $5 \mathrm{H}, 6-82$ & 43.9 & 2.94 & 2.50 & 423 & 239 & 203 & 1.23 & 1.46 & 0.23 & 6.49 & -25.75 & 10.98 \\
\hline $5 \mathrm{H}, 6-102$ & 44.1 & 3.84 & 3.35 & 424 & 261 & 228 & 1.47 & 1.47 & 0.26 & 5.65 & -25.42 & 10.20 \\
\hline $5 \mathrm{H}, 7-2$ & 44.31 & 2.75 & 3.41 & 428 & 233 & 289 & 1.18 & 1.40 & 0.21 & 6.64 & -24.64 & 12.37 \\
\hline $5 \mathrm{H}, 7-22$ & 44.51 & 3.35 & 2.63 & 425 & 234 & 184 & 1.43 & 1.50 & 0.25 & 5.95 & -26.75 & 9.60 \\
\hline $5 \mathrm{H}, 7-42$ & 44.71 & 2.76 & 3.65 & 423 & 265 & 225 & 1.35 & 1.39 & 0.20 & 6.93 & -27.01 & 11.03 \\
\hline $6 \mathrm{H}, 1-2$ & 45.63 & 4.96 & 2.08 & 423 & 362 & 152 & 1.37 & 1.66 & 0.29 & 5.84 & -27.68 & 6.94 \\
\hline $6 \mathrm{H}, 1-22$ & 45.83 & 3.35 & 1.97 & 423 & 282 & 166 & 1.19 & 1.61 & 0.26 & 6.17 & -25.66 & 8.41 \\
\hline $6 \mathrm{H}, 1-42$ & 46.03 & 2.99 & 1.63 & 420 & 282 & 154 & 1.06 & 1.29 & 0.24 & 5.41 & -27.02 & 9.43 \\
\hline $6 \mathrm{H}, 1-62$ & 46.23 & 3.88 & 2.32 & 419 & 268 & 160 & 1.45 & 1.58 & 0.27 & 5.91 & -26.20 & 8.30 \\
\hline $6 \mathrm{H}, 1-82$ & 46.43 & 4.16 & 2.15 & 420 & 275 & 142 & 1.51 & 1.73 & 0.27 & 6.35 & -26.79 & 8.36 \\
\hline $6 \mathrm{H}, 1-102$ & 46.63 & 5.02 & 2.18 & 407 & 356 & 155 & 1.41 & 1.72 & 0.28 & 6.19 & -27.92 & 9.93 \\
\hline $6 \mathrm{H}, 1-122$ & 46.83 & 3.85 & 2.17 & 419 & 285 & 161 & 1.35 & 1.62 & 0.27 & 6.09 & -26.66 & 8.91 \\
\hline $6 \mathrm{H}, 2-2$ & 47.01 & 3.79 & 3.26 & 426 & 260 & 223 & 1.46 & 1.73 & 0.23 & 7.57 & -26.33 & 7.18 \\
\hline $6 \mathrm{H}, 2-22$ & 47.21 & 4.19 & 2.21 & 414 & 325 & 171 & 1.29 & 1.59 & 0.30 & 5.23 & -26.83 & 7.89 \\
\hline $6 \mathrm{H}, 2-42$ & 47.41 & 4.16 & 1.98 & 414 & 313 & 149 & 1.33 & 1.58 & 0.29 & 5.49 & -26.87 & 8.25 \\
\hline $6 \mathrm{H}, 2-52$ & 47.51 & 4.46 & 1.81 & 411 & 323 & 131 & 1.38 & 1.56 & 0.31 & 5.07 & -27.45 & 7.64 \\
\hline $6 \mathrm{H}, 2-62$ & 47.61 & 4.19 & 2.09 & 410 & 313 & 156 & 1.34 & 1.54 & 0.29 & 5.22 & -26.49 & 8.77 \\
\hline $6 \mathrm{H}, 2-82$ & 47.81 & 3.78 & 2.05 & 417 & 282 & 153 & 1.34 & 1.59 & 0.30 & 5.37 & -26.85 & 7.82 \\
\hline $6 \mathrm{H}, 2-102$ & 48.01 & 4.18 & 1.98 & 417 & 303 & 143 & 1.38 & 1.62 & 0.28 & 5.79 & -26.37 & 8.02 \\
\hline $6 \mathrm{H}, 3-2$ & 48.15 & 4.11 & 2.82 & 423 & 270 & 186 & 1.52 & 1.69 & 0.25 & 6.67 & -25.26 & 8.11 \\
\hline $6 \mathrm{H}, 3-22$ & 48.35 & 4.21 & 2.10 & 423 & 312 & 156 & 1.35 & 1.57 & 0.28 & 5.66 & -26.04 & 10.25 \\
\hline $6 \mathrm{H}, 3-42$ & 48.55 & 3.75 & 1.94 & 415 & 365 & 196 & 1.49 & 1.48 & 0.28 & 5.37 & -26.38 & 9.71 \\
\hline $6 \mathrm{H}, 3-62$ & 48.75 & 3.80 & 2.87 & 415 & 268 & 202 & 1.42 & 1.51 & 0.30 & 4.97 & -26.69 & 7.37 \\
\hline $6 \mathrm{H}, 3-82$ & 48.95 & 3.56 & 1.76 & 413 & 330 & 163 & 1.08 & 1.47 & 0.30 & 4.83 & -26.66 & 7.55 \\
\hline $6 \mathrm{H}, 3-102$ & 49.15 & 3.67 & 1.75 & 413 & 331 & 158 & 1.11 & 1.42 & 0.26 & 5.40 & -27.28 & 8.56 \\
\hline $6 \mathrm{H}, 3-122$ & 49.35 & 4.03 & 2.22 & 419 & 303 & 167 & 1.33 & 1.60 & 0.26 & 6.10 & -26.17 & 10.57 \\
\hline $6 \mathrm{H}, 4-2$ & 49.65 & 3.99 & 3.56 & 428 & 424 & 216 & 1.65 & 1.84 & 0.27 & 6.75 & -24.96 & 9.97 \\
\hline $6 \mathrm{H}, 4-22$ & 49.85 & 3.89 & 2.70 & 421 & 258 & 179 & 1.51 & 1.74 & 0.25 & 6.93 & -25.87 & 8.99 \\
\hline $6 \mathrm{H}, 4-38$ & 50.01 & 3.33 & 2.76 & 421 & 241 & 200 & 1.38 & 1.56 & 0.23 & 6.73 & -25.45 & 8.16 \\
\hline $6 \mathrm{H}, 4-42$ & 50.05 & 3.50 & 2.58 & 421 & 243 & 179 & 1.44 & 1.59 & 0.25 & 6.28 & -25.75 & 8.94 \\
\hline $6 \mathrm{H}, 4-62$ & 50.25 & 4.31 & 2.45 & 420 & 276 & 157 & 1.56 & 1.77 & 0.26 & 6.80 & -26.69 & 8.55 \\
\hline $6 \mathrm{H}, 4-82$ & 50.45 & 4.13 & 2.73 & 421 & 279 & 184 & 1.48 & 1.72 & 0.25 & 6.90 & -25.75 & 7.83 \\
\hline $6 \mathrm{H}, 4-102$ & 50.65 & 3.28 & 3.29 & 422 & 258 & 259 & 1.27 & 1.44 & 0.27 & 5.37 & -25.60 & 11.24 \\
\hline $6 \mathrm{H}, 4-122$ & 50.85 & 3.57 & 2.81 & 420 & 232 & 182 & 1.54 & 1.64 & 0.24 & 6.81 & -25.53 & 9.59 \\
\hline $6 \mathrm{H}, 5-2$ & 51.15 & 4.17 & 2.99 & 423 & 273 & 195 & 1.53 & 1.68 & 0.24 & 7.01 & -24.92 & 5.95 \\
\hline $6 \mathrm{H}, 5-22$ & 51.35 & 3.77 & 3.09 & 423 & 290 & 238 & 1.3 & 1.37 & 0.20 & 6.76 & -26.02 & 7.96 \\
\hline $6 \mathrm{H}, 5-42$ & 51.55 & 3.91 & 3.14 & 426 & 254 & 204 & 1.54 & 1.69 & 0.26 & 6.42 & -26.05 & 10.51 \\
\hline $6 \mathrm{H}, 5-62$ & 51.75 & 3.31 & 2.12 & 422 & 242 & 155 & 1.37 & 1.54 & 0.23 & 6.57 & -25.76 & 9.56 \\
\hline $6 \mathrm{H}, 5-82$ & 51.95 & 3.91 & 3.06 & 424 & 266 & 208 & 1.47 & 1.68 & 0.25 & 6.72 & -25.95 & 9.25 \\
\hline $6 \mathrm{H}, 5-102$ & 52.15 & 4.45 & 3.21 & 423 & 273 & 197 & 1.63 & 1.84 & 0.26 & 7.01 & -25.73 & 9.64 \\
\hline
\end{tabular}


Table T1 continued.

\begin{tabular}{|c|c|c|c|c|c|c|c|c|c|c|c|c|}
\hline \multirow[b]{3}{*}{$\begin{array}{l}\text { Core, section, } \\
\text { interval }(\mathrm{cm})\end{array}$} & \multirow[b]{3}{*}{$\begin{array}{l}\text { Depth } \\
\text { (mbsf) }\end{array}$} & \multicolumn{6}{|c|}{ Rock-Eval pyrolysis } & \multirow{2}{*}{\multicolumn{3}{|c|}{ Major elements }} & \multirow{2}{*}{\multicolumn{2}{|c|}{ Isotopes }} \\
\hline & & & & & & Ol & & & & & & \\
\hline & & $\begin{array}{c}\text { (mg HC/g } \\
\text { rock) }\end{array}$ & $\underset{\text { rock) }}{\left(\mathrm{mg} \mathrm{CO}_{2} / g\right.}$ & $\begin{array}{l}T_{\max } \\
\left({ }^{\circ} \mathrm{C}\right)\end{array}$ & $\begin{array}{c}(\mathrm{mg} \mathrm{HC} / \mathrm{g} \\
\mathrm{TOC})\end{array}$ & $\begin{array}{c}\left(\mathrm{mg} \mathrm{CO}_{2} / \mathrm{g}\right. \\
\mathrm{TOC})\end{array}$ & $\begin{array}{l}\text { TOC } \\
\text { (wt\%) }\end{array}$ & $\begin{array}{c}\text { TOC } \\
\text { (wt\%) }\end{array}$ & $\begin{array}{c}\mathrm{TN} \\
(\mathrm{wt} \%)\end{array}$ & $\begin{array}{l}\mathrm{C} / \mathrm{N} \\
\text { ratio }\end{array}$ & $\begin{array}{l}\delta^{13} \mathrm{C} \\
(\% 0)\end{array}$ & $\begin{array}{l}\delta^{15} N \\
(\% o)\end{array}$ \\
\hline $6 \mathrm{H}, 5-122$ & 52.35 & 4.50 & 3.26 & 422 & 304 & 220 & 1.48 & 1.67 & 0.26 & 6.34 & -25.90 & 9.83 \\
\hline $6 \mathrm{H}, 5-138$ & 52.51 & 4.41 & 3.49 & 410 & 298 & 236 & 1.48 & 1.73 & 0.26 & 6.78 & -26.08 & 9.15 \\
\hline $6 \mathrm{H}, 6-2$ & 52.65 & 4.47 & 2.89 & 423 & 274 & 177 & 1.63 & 1.87 & 0.25 & 7.37 & -25.82 & 7.76 \\
\hline $6 \mathrm{H}, 6-22$ & 52.85 & 4.42 & 2.93 & 423 & 273 & 181 & 1.62 & 1.80 & 0.26 & 7.02 & -25.45 & 8.71 \\
\hline $6 \mathrm{H}, 6-42$ & 53.05 & 5.07 & 3.18 & 423 & 345 & 216 & 1.47 & 1.88 & 0.26 & 7.18 & -25.99 & 8.60 \\
\hline $6 \mathrm{H}, 6-62$ & 53.25 & 3.79 & 2.72 & 424 & 241 & 173 & 1.57 & 1.62 & 0.26 & 6.19 & -25.07 & 8.74 \\
\hline $6 \mathrm{H}, 6-82$ & 53.45 & 4.04 & 2.76 & 423 & 259 & 177 & 1.56 & 1.77 & 0.26 & 6.91 & -25.99 & 8.07 \\
\hline $6 \mathrm{H}, 6-102$ & 53.65 & 4.10 & 3.73 & 423 & 283 & 257 & 1.45 & 1.62 & 0.25 & 6.51 & -25.30 & 9.95 \\
\hline $6 \mathrm{H}, 6-122$ & 53.85 & 3.83 & 2.64 & 425 & 264 & 182 & 1.45 & 1.68 & 0.27 & 6.32 & -25.26 & 9.45 \\
\hline $6 \mathrm{H}, 7-2$ & 54.15 & 4.57 & 2.66 & 429 & 326 & 190 & 1.4 & 1.70 & 0.23 & 7.35 & -25.24 & 6.79 \\
\hline $6 \mathrm{H}, 7-22$ & 54.35 & 3.60 & 3.15 & 424 & 252 & 220 & 1.43 & 1.66 & 0.26 & 6.40 & -25.33 & 10.06 \\
\hline
\end{tabular}

$\mathrm{HC}=$ hydrocarbon, $\mathrm{HI}=$ hydrogen index, $\mathrm{OI}=$ oxygen index, $\mathrm{TOC}=$ total organic carbon, $\mathrm{TN}=$ total nitrogen. 